\title{
Nanoparticle-based delivery systems modulate the tumor microenvironment in pancreatic cancer for enhanced therapy
}

Ming Jia ${ }^{1 \dagger}$, Dan Zhang ${ }^{2 \dagger}$, Chunxiang Zhang ${ }^{3 *}$ and Chunhong $\mathrm{Li}^{{ }^{*}}$

\begin{abstract}
Pancreatic cancer is one of the most lethal malignant tumors with a low survival rate, partly because the tumor microenvironment (TME), which consists of extracellular matrix (ECM), cancer-associated fibroblasts (CAFs), immune cells, and vascular systems, prevents effective drug delivery and chemoradiotherapy. Thus, modulating the microenvironment of pancreatic cancer is considered a promising therapeutic approach. Since nanoparticles are one of the most effective cancer treatment strategies, several nano-delivery platforms have been developed to regulate the TME and enhance treatment. Here, we summarize the latest advances in nano-delivery systems that alter the TME in pancreatic cancer by depleting ECM, inhibiting CAFs, reversing immunosuppression, promoting angiogenesis, or improving the hypoxic environment. We also discuss promising new targets for such systems. This review is expected to improve our understanding of how to modulate the pancreatic cancer microenvironment and guide the development of new therapies.
\end{abstract}

Keywords: Pancreatic cancer, Tumor microenvironment, Nano-delivery systems, Cancer-associated fibroblasts, Extracellular matrix, Immunosuppression

\footnotetext{
*Correspondence: zhangchx999@163.com; lispringhong@126.com

${ }^{\dagger}$ Ming Jia and Dan Zhang contributed equally to this work

${ }^{1}$ Department of Pharmaceutical Sciences, School of Pharmacy,

Southwest Medical University, No.1, Section 1, Xianglin Road, Luzhou,

Sichuan 646000, People's Republic of China

${ }^{3}$ The Key Laboratory of Medical Electrophysiology of the Ministry

of Education, Southwest Medical University, No.1, Section 1, Xianglin

Road, Luzhou, Sichuan 646000, People's Republic of China

Full list of author information is available at the end of the article
}

(c) The Author(s) 2021. Open Access This article is licensed under a Creative Commons Attribution 4.0 International License, which permits use, sharing, adaptation, distribution and reproduction in any medium or format, as long as you give appropriate credit to the original author(s) and the source, provide a link to the Creative Commons licence, and indicate if changes were made. The images or other third party material in this article are included in the article's Creative Commons licence, unless indicated otherwise in a credit line to the material. If material is not included in the article's Creative Commons licence and your intended use is not permitted by statutory regulation or exceeds the permitted use, you will need to obtain permission directly from the copyright holder. To view a copy of this licence, visit http://creativecommons.org/licenses/by/4.0/. The Creative Commons Public Domain Dedication waiver (http://creativeco mmons.org/publicdomain/zero/1.0/) applies to the data made available in this article, unless otherwise stated in a credit line to the data. 


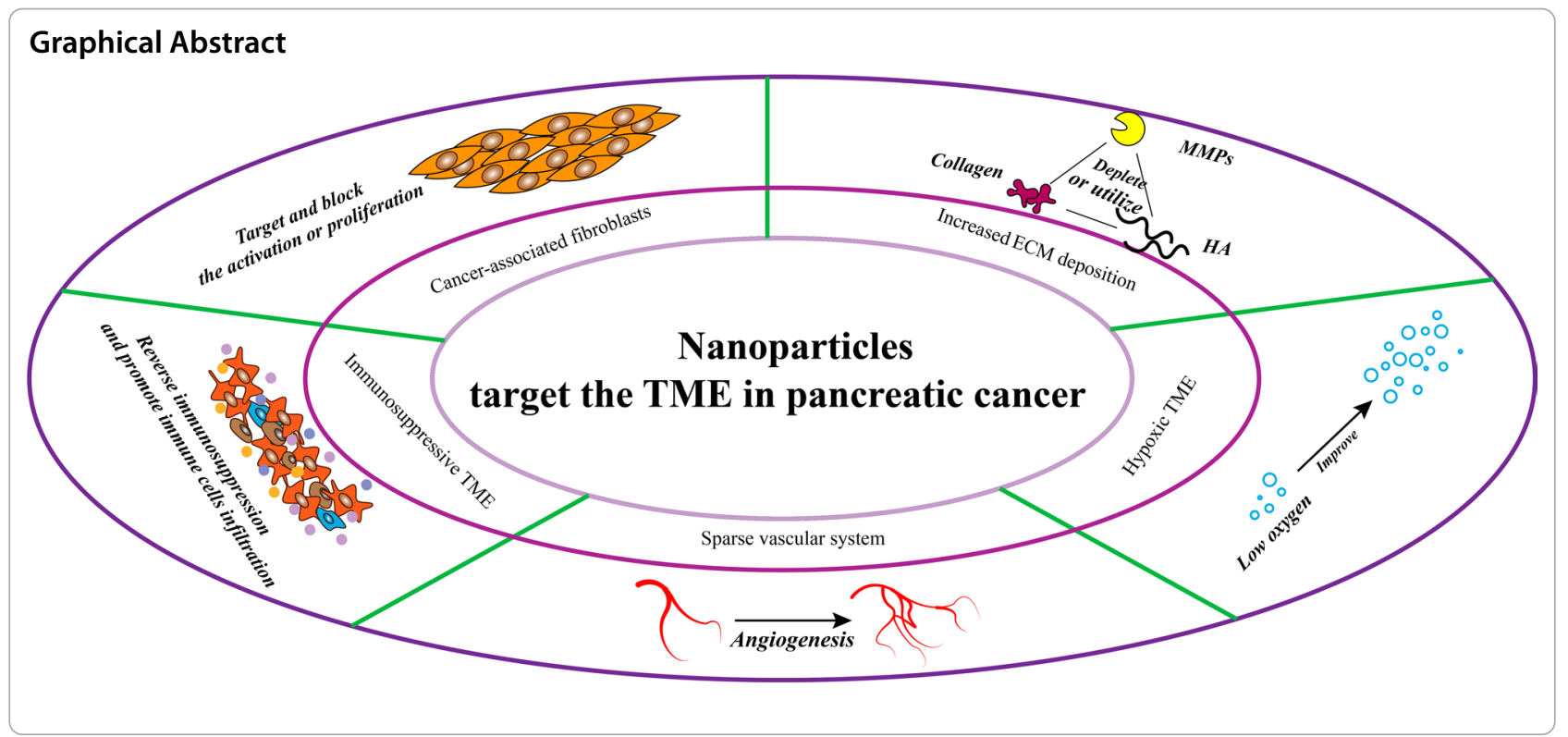

\section{Introduction}

Pancreatic cancer is one of the most aggressive and fatal malignant tumors, with a five-year survival rate of less than $5 \%$. Fully $90 \%$ of cases occur as pancreatic ductal adenocarcinoma (PDAC). This cancer type progresses rapidly, it responds weakly to treatment, and patient prognosis is poor. In addition, metastasis reduces survival to less than half a year [1-4]. There are no biomarkers specific for pancreatic cancer, and biomarkers for other cancers do not recognize it reliably $[5,6]$. Pancreatic cancer cells can adapt gradually to the nutrient-deficient environment induced by the desmoplastic reaction and develop stem cell-like properties to meet their energy needs [7-9]. Excessive stroma caused by desmoplastic reaction is the main feature of pancreatic cancer TME that is rarely observed in other malignant tumors [10]. Dense fibrous stroma tightly surrounds the cancer cells $[11,12]$, one of the defining histopathological features of pancreatic cancer, which constitutes more than $80 \%$ of the tumor mass [13] and is much higher than that of other tumors [14]. On the one hand, the fibrous stroma is so dense that the vasculature becomes extremely sparse, resulting in anti-angiogenic therapy that is suitable for most tumors but not for pancreatic cancer [15]. On the other hand, unlike other tumors, the tumor stroma of pancreatic cancer seems to act as a natural barrier between the body's immune system and the tumor, limiting the use of immunotherapy $[16,17]$. Most importantly, the barrier protects tumor cells from being attacked by conventional chemotherapy drugs to develop chemoresistance [18]. Therefore, when designing therapeutic strategies for pancreatic cancer, we have to consider the unique TME (Fig. 1).

Various nano-delivery systems have been developed and widely used for diagnosing and treating various cancers [19, 20]. Nano-delivery systems can increase biocompatibility and solubility and prolong time in circulation [21], while their facile modification and multifunctional properties enable further optimization, such as for targeting particular cells. The ischemic and hypoxic TME consists of ECM components, as well as cells and cytokines closely related to growth, invasion, and metastasis [22, 23]. Nano-delivery systems have been developed to act on these components or stimulate certain changes in order to inhibit the survival and growth of pancreatic cancer cells.

\section{Extracellular matrix (ECM)}

The ECM is a dense network that consists of collagen, fibronectin, proteoglycan, hyaluronic acid (HA), catalytic enzymes, and proteases. It is is present in all tissues, where it helps maintain structure and biochemistry [24, 25]. All ECM components interact closely with various cells, forming a dynamic microsite with various functional states [26]. In contrast to other tumors [27-29], the increased ECM deposition in pancreatic cancer inhibits immune cell infiltration and drug delivery to the tumor core [30]. For example, only $0.7 \%$ of the administered nanoparticle dose can be delivered to the solid tumor site [31]. Abnormally overexpressed matrix components may lead to strong invasive metastasis, while maintaining a specific environment that promotes cancer cell proliferation [32,33]. This connective tissue hyperplasia is very 

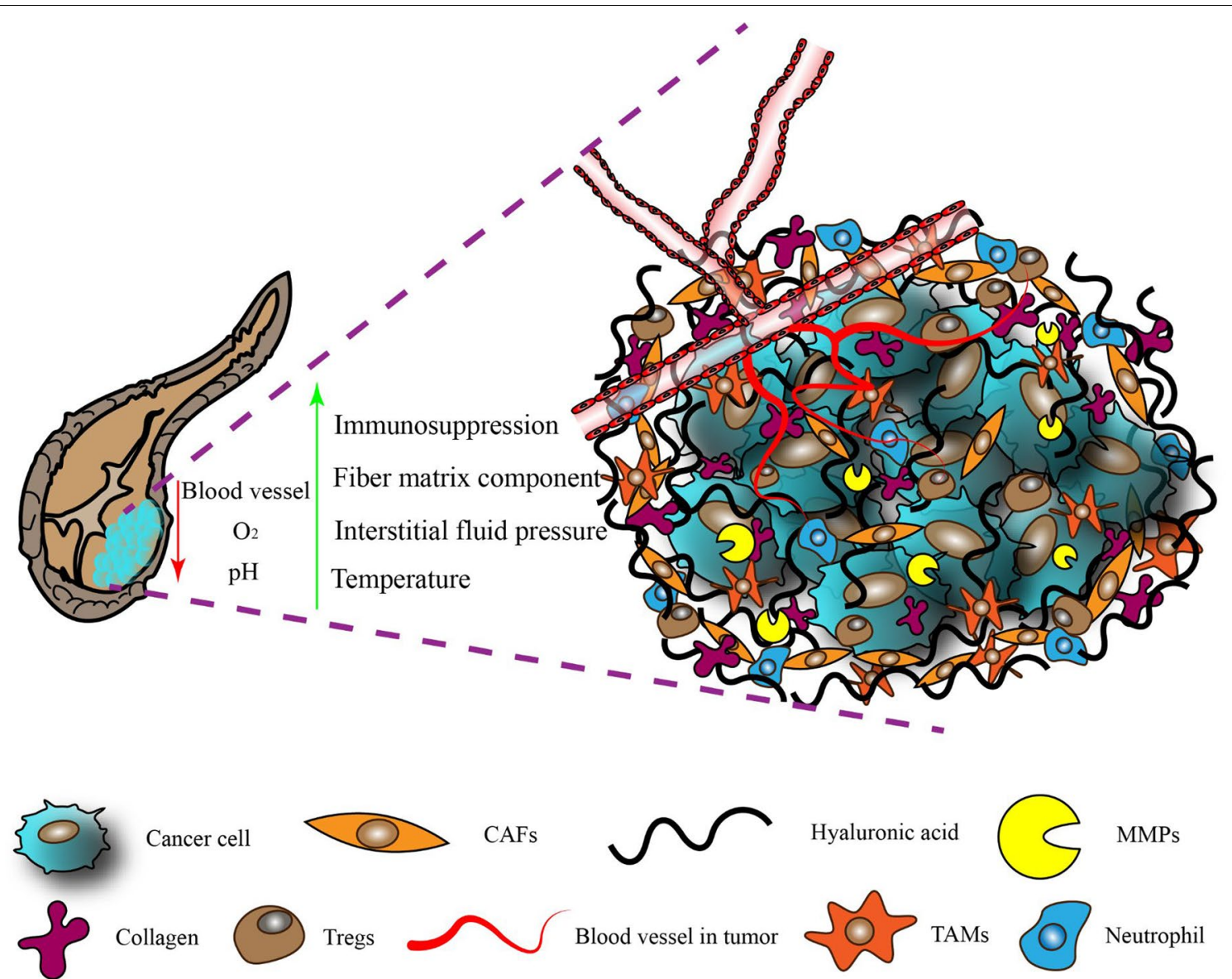

Fig. 1 The tumor microenvironment (TME) in pancreatic cancer. The expanded extracellular matrix increases interstitial stress, while collapse of blood vessels reduces oxygen levels and acidifies the $\mathrm{pH}$. Low infiltration by immune cells and their phenotypic transformation inhibit anti-tumor immune responses. Various activation pathways generate cancer-associated fibroblasts (CAFs), key cellular components of the TME. MMPs, matrix metalloproteinases; TAMs, tumor-associated macrophages; Tregs, regulatory T cells

important for the pathogenesis of pancreatic cancer, indicating that ECM modulation may be a promising treatment strategy for this type of cancer (Fig. 2).

\section{Hyaluronic acid (HA)}

HA, a key component of the ECM, is a hydrophilic glucosamine polysaccharide that is strongly expressed in pancreatic tumors. It reduces tumor perfusion and infiltration by anti-tumor immune cells, while increasing tumor interstitial fluid pressure and vascular collapse [34]. HA has been closely associated with tumor proliferation and metastasis [35]. Median overall survival of pancreatic cancer patients is 15 months shorter among those showing high HA deposition than among those with low deposition [36]. This suggests that HA accumulation is one of the main factors for poor prognosis in patients with pancreatic cancer.

Hyaluronidase (HAase) is an endogenous degradation enzyme that quickly transforms HA into oligosaccharides and low-molecular-weight HA. Studies in genetically engineered mouse models of PDAC showed that combining gemcitabine with PEGylated recombinant human HAase PH20 (PEGPH20), which can rapidly and sustainably deplete HA, can increase tumor perfusion and vascular permeability, even more so than gemcitabine alone [37]. In fact, this combination therapy has proven effective even against stage IV pancreatic cancer involving high HA deposition [9]. These results indicate that HA depletion can promote drug delivery to the tumor site. However, HAase can be inactivated and degraded in vivo, reducing its half-life in serum and limiting its accumulation at the tumor site.

Therefore, nanoparticles (NPs) have been used to protect the enzyme from side effects and improve its tumor delivery. For instance, recombinant human HAase PH20 ( $\mathrm{rHuPH} 20)$ was loaded on the surface of poly(lactic-coglycolic acid)- $b$-polyethylene glycol (PLGA-PEG) NPs, which were then coated with an additional PEG layer to 


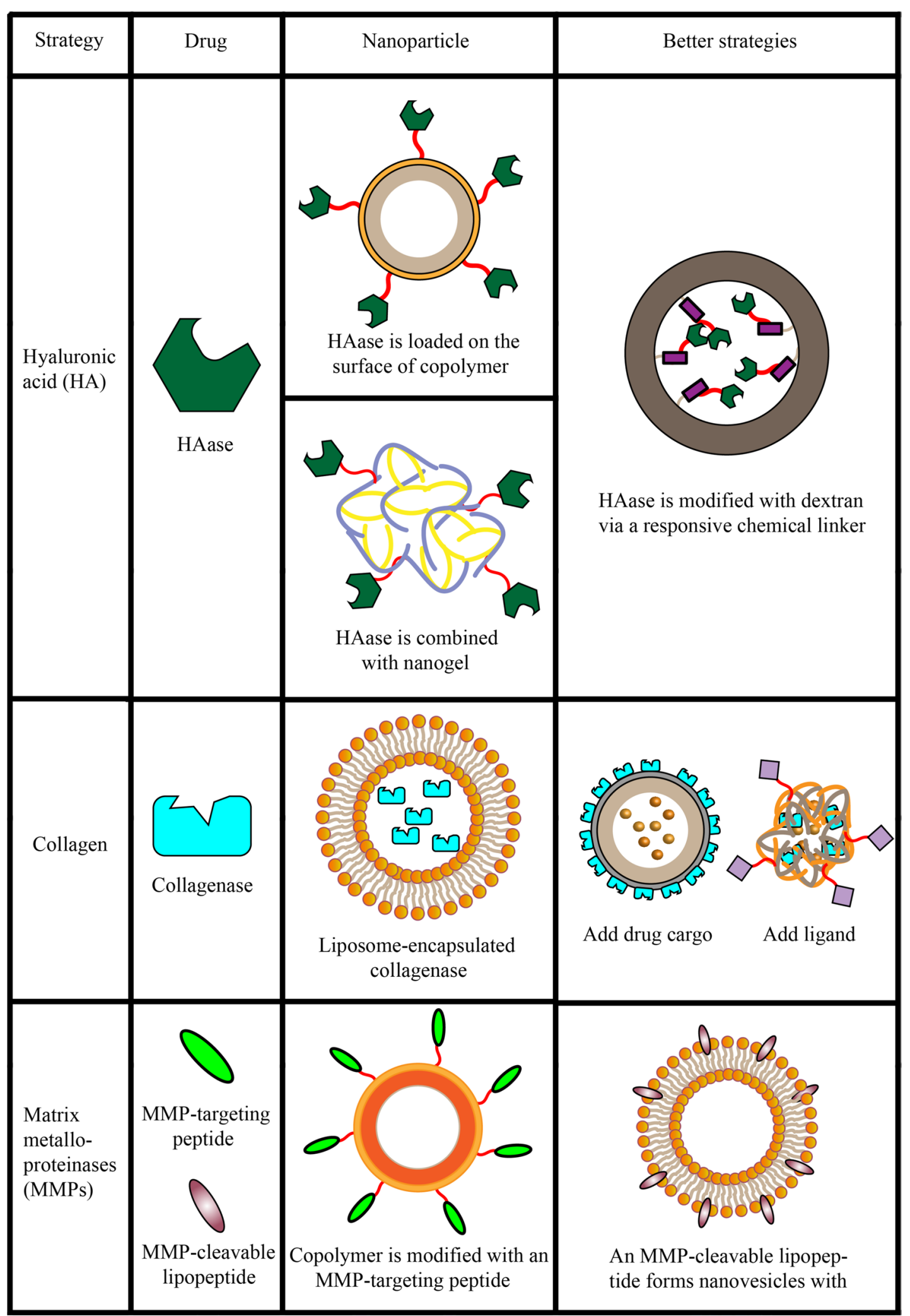

Fig. 2 Nanoparticle delivery systems used to target the extracellular matrix in the tumor microenvironment 
protect the $\mathrm{rHuPH} 20$ and improve its accumulation at the tumor site [38]. In another study, HAase was chemically modified to produce thiols, and this enzyme was combined with nanogels for protection and improved delivery at the tumor site [39]. However, those previous studies conjugated linear PEG chains to HAase via noncleavable bonds, which can affect enzyme activity. Thus, HAase was modified with dextran via a chemical linker, which could dissociate within the acidic TME, restoring the enzyme's activity [40]. Clinical studies confirmed the ability of HAase to promote drug absorption and penetration in tumor tissues expressing high HA [26, 41].

\section{Collagen}

Collagen is another major component of ECM that is involved in the fibrosis of the pancreatic cancer microenvironment. Excessive production of collagen can lead to drug resistance and limit drug absorption [42]. Analogously to HA depletion from ECM, collagen catabolism has been explored as a way to increase drug delivery [43, 44], and collagenase has been approved by the U.S. Food and Drug Administration and the European Medicines Agency for the treatment of palm fibromatosis [45]. For instance, the efficacy of chemotherapy in PDAC mice was significantly increased by pretreatment with liposomeencapsulated collagenase, indicating that collagenase can enhance tumor penetration and chemotherapy [46]. However, long intervals between chemotherapeutic drug administrations may prevent its timely delivery to the tumor site, leading to ECM destruction that may further promote cancer metastasis [47]. To avoid this risk and improve drug efficacy, collagenase and doxorubicin (DOX) were co-loaded onto PLGA NPs carrying an adhesive polydopamine layer [48]. In another approach, the monoclonal anti-HER2 antibody trastuzumab and collagenase have been formulated into a thermosensitive PLGA-PEG-PLGA hydrogel to improve drug release and treatment efficacy [49]. Nevertheless, collagenase has been applied in cancer treatment much less often than HAase, probably because its cleavage products cannot be effectively separated from collagen fibers, leading only to small local changes in the collagen structure [50].

\section{Matrix metalloproteinases (MMPs)}

ECM also contains an abundance of proteolytic enzymes that help maintain its structure. Among them, matrix metalloproteinases (MMPs) are known to play a key role in cancer occurrence, tumor growth, and metastasis. Studies have shown that MMP-1, MMP-2, MMP-7, MMP-9, membrane type 1-MMP (MT1-MMP), MT2MMP, and MT3-MMP are overexpressed in pancreatic cancer tumors, while MMP-2, MMP-7, and MMP-9 have been identified as potential biomarkers of pancreatic cancer [51]. NPs modified with a MT1-MMP-binding peptide showed excellent targeting and uptake ability in a pancreatic cancer mouse model [52]. In another study, an MMP-9-cleavable lipopeptide was synthesized to form nanovesicles with various lipids [53]. In the presence of elevated glutathione levels, the outer PEG groups were reductively removed, exposing the substrate lipopeptides to MMP-9. As a result, the lipid bilayer of the vesicle was disrupted, releasing the encapsulated content. In this way, MMP-9-responsive nanovesicles were able to control tumor growth more effectively than MMP-9-free vesicles, suggesting that MMPs can be used as an inducible "trigger" to enhance drug accumulation in pancreatic tumors.

\section{Cancer-associated fibroblasts (CAFs)}

In normal pancreatic tissue, fibroblasts and pancreatic stellate cells are responsible for maintaining the normal structure of the glandular connective tissue [54]. However, in cancer tissues, various activation pathways generate CAFs from bone marrow mesenchymal stem cells, pancreatic stellate cells, and resting fibroblasts. These activation pathways include sonic hedgehog (SHH), transforming growth factor- $\beta$ (TGF- $\beta$ ), tumor necrosis factor- $\alpha$ (TNF- $\alpha$ ), interleukin (IL)-1, IL-6, and IL-10. CAFs are a key component of the tumor stroma, where they secrete molecules and contract [55-58]. They are densely arranged around all pancreatic tumor sites, while also appearing in some benign tissues and ducts [59-61]. Therefore, blocking the activation and proliferation of CAFs or targeting them for drug delivery may serve as a new therapeutic strategy for pancreatic cancer (Fig. 3).

Recently, CAF-targeting biodegradable polymer NPs loaded with $\alpha$-mangostin and coated with CREKA peptide were developed [62]. $\alpha$-Mangostin is known to modulate the TME by interfering with the TGF- $\beta$ / Smad signaling pathway and blocking the activation of CAFs, while the CREKA peptide has specific affinity for fibronectin, which is overexpressed on the CAF membrane. The peptide coating enhanced the uptake of CAFs, while the combination with $\alpha$-mangostin strengthened CAF inhibition, reshaping the TME in a way that compromised the stroma barrier. Similarly, the SHH inhibitor cyclopamine has been encapsulated into PLGA NPs, which were coated with erythrocyte membrane for better biocompatibility and longer circulation time [63].

However, blocking only the activation of CAFs may not effectively kill pancreatic tumors. Therefore, cyclopamine, which can deplete stroma-producing CAFs, has been encapsulated into polymer micelles together with paclitaxel (PTX), an effective inhibitor of tumor proliferation [64]. Compared to the effects of using cyclopamine alone, co-delivery of cyclopamine and a 


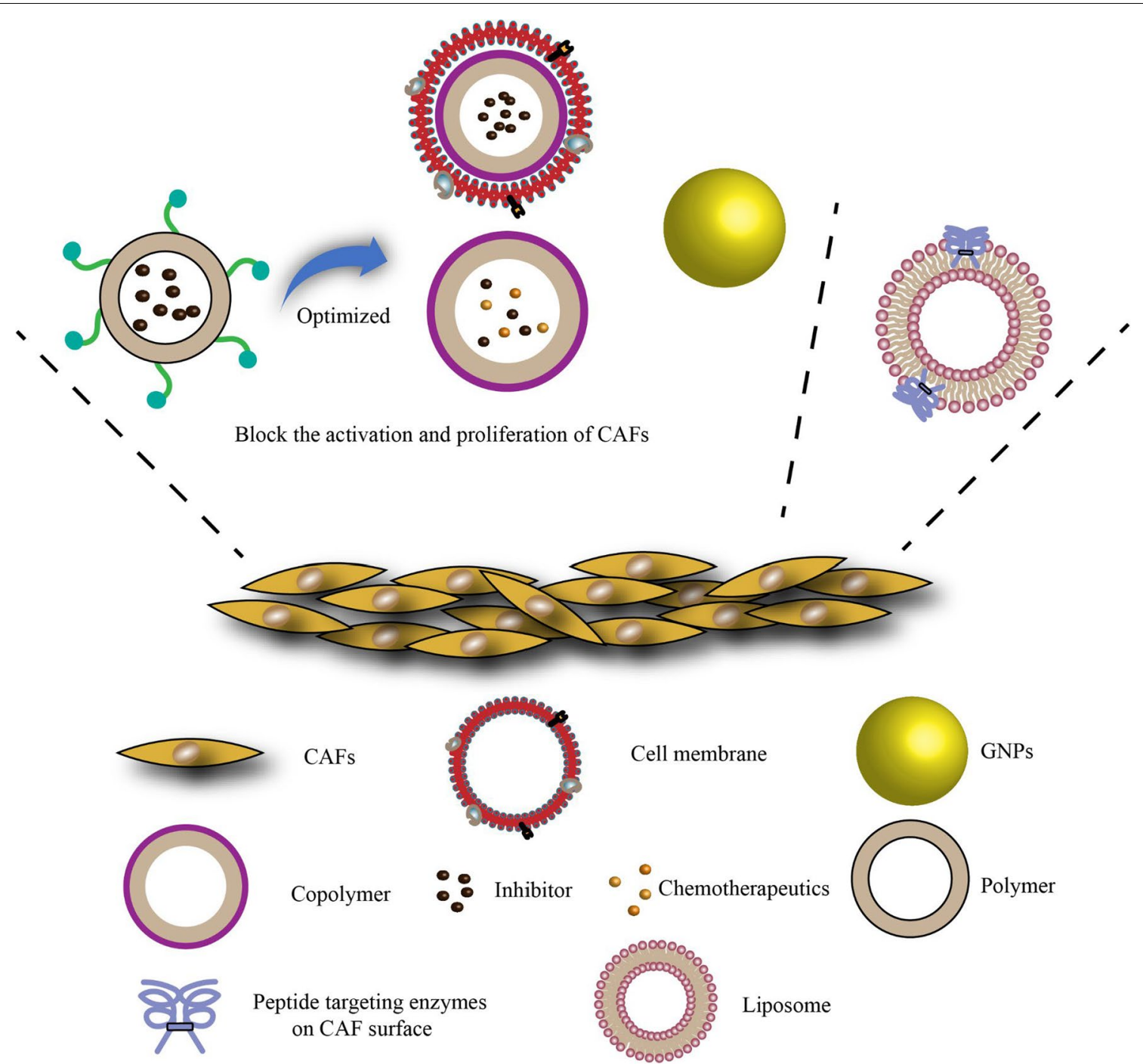

Fig. 3 Different nanoparticle optimization strategies used to block the activation and proliferation of cancer-associated fibroblasts (CAFs). GNPS, gold nanoparticles

chemotherapeutic drug reduced the adverse effects of stroma ablation and led to greater therapeutic effect, which could be optimized by regulating the ratio of the two agents.

Enzymes overexpressed on the CAF membrane have also been targeted in order to optimize drug delivery. For instance, the albumin nanoparticle of PTX (HSA-PTX) was encapsulated into cleavable amphiphilic peptide (CAP)-modified liposomes that were responsive to fibroblast-activated protein- $\alpha$ (FAP- $\alpha$ ), a membrane-bound serine protease specifically expressed on the surface of CAFs. CAP enhanced drug accumulation at the tumor site and promoted the enzymatic reaction with FAP- $\alpha$, thus facilitating the release of HSA-PTX [56].
Interestingly, the materials used to prepare nanocarriers themselves can inhibit CAF activation. For example, gold NPs (GNPs) have been used to shift CAF phenotype from activated to stationary by inducing endogenous lipid synthesis [65], suggesting that GNPs may enable the development of functional drug nanodelivery strategies.

\section{Immunosuppressive TME}

During the transformation of normal pancreatic tissues into cancer tissues, connective tissue hyperplasia leads to tissue fibrosis, while the numbers of tumor-associated macrophages (TAMs) of the M2 phenotype, neutrophils of the N2 phenotype, and regulatory $\mathrm{T}$ cells (Tregs) increase, changing the immunophenotype of the disease [54]. TAMs 
are one of the most abundant immune cells in the TME [66], and the ratio of TAMs with the M2 phenotype to the number with the M1 phenotype is inversely related to disease progression and survival time [67]. In the presence of T-helper 2 cell-type cytokines, M1 TAMs are polarized toward the M2 phenotype, which is immunosuppressive and promotes tumor growth [68]. In addition, the interaction of M2 TAMs with cancer cells and various non-cancer cells promotes cancer cell proliferation, drug resistance, and distant metastasis, attracting macrophages that form malignant feedback loops and continuously strengthen immunosuppression [69].

Ly6C, a mouse homolog of CD59, is highly expressed in TAMs. To specifically target Ly6C-overexpressing TAMs, the surface of porous silicon nanocarriers was modified with an anti-Ly6C antibody (Fig. 4a) [70]. Another study showed that the polarization of M2 TAMs is mediated by multiple signaling pathways [69], especially PI3K- $\gamma$ and CSF-1/CSF-1R. Therefore, a nanomicelle carrying an M2 TAM-targeting peptide was prepared to co-deliver the PI3K- $\gamma$ inhibitor BEZ 235 and an siRNA silencing the colony stimulating factor-1 receptor (CSF-1R) in order to inhibit TAMs specifically [71]. The nanomicelle inhibited both the PI3k- $\gamma$ and CSF-1R pathways, while the level of M2 TAMs decreased and that of M1 TAMs increased, resulting in a remodelling of the immune microenvironment. In another therapeutic approach, NPs encapsulating 5 '-triphosphate double-stranded RNA (ppp dsRNA) were developed. Lipid-calcium phosphate NPs were selected as the drug delivery vector, since they have been widely used to deliver phosphorylated biomolecules, while modified aminoethyl anisamide was used as the targeted ligand. The delivered ppp dsRNA induced cancer cell apoptosis by binding to retinoic acid-inducible gene I-like receptors to produce type I interferon and silence $\mathrm{Bcl} 2$, thus increasing the proportion of M1 TAMs and reducing immunosuppression in the TME [72].

Interfering with the indoleamine 2,3-dioxygenase (IDO) pathway to reverse the immunosuppressive TME has also been shown to enhance immunogenic cell death (ICD). Specifically, IDO1 siRNA encapsulated in lipid NPs reached the tumor site without excessive loss, where it downregulated Tregs to promote ICD (Fig. 4b) [73]. In a subsequent study, a more effective co-delivery nanosystem was prepared, where the ICD inducer oxaliplatin and the IDO inhibitor indomethacin were co-encapsulated in mesoporous silica NPs coated with lipid bilayers, achieving greater synergy than the previous method (Fig. 4c) [74]. These results illustrate how incorporating anti-immunosuppression strategies into NP-based drug delivery can improve therapeutic efficacy.

\section{Angiogenesis}

Anti-angiogenesis therapies are commonly used in various cancers, including glioblastoma, non-small cell lung cancer, renal cell carcinoma, hepatocellular carcinoma, and multiple myeloma [15, 24]. However, no clinically effective anti-angiogenesis agents have been reported for pancreatic cancer, as its vascular system is affected by high interstitial fluid pressure and the microvessel density is inversely related to the interstitial surface area [24].

Cilengitide, a small molecule with strong anti-angiogenesis effects, induces endothelial cell apoptosis at micromolar concentrations, but it promotes endothelial cell migration and enhances tumor angiogenesis at nanomolar concentrations [75]. To improve its tumortargeting ability, cilengitide-loaded nanocarriers were prepared. High cilengitide loading led to high affinity and internalization, inhibiting angiogenesis and improving the ability of chemotherapy drugs to kill endothelial cells. Low cilengitide loading prevented integrin-mediated endocytosis, but did not promote endothelial cell migration due to insufficient interaction of the drug with integrins [76-78].

Cilengitide was encapsulated in doxorubicin (DOX)loaded thermosensitive liposomes via an MT1-MMPcleavable peptide (MC-T-DOX). After reaching the tumor site, cilengitide was released through MT1MMP cleavage on tumor endothelial cells (Fig. 5). This approach prevented excessive endothelial cell endocytosis and ensured sufficient interaction of cilengitide with $\alpha_{v} \beta_{3}$ integrins on endothelial cells to form new blood vessels into ischemic areas, thereby improving the delivery of drug-loaded liposomes [79]. Compared with the previously mentioned ECM depletion strategy $[37,38,46]$, which cannot be strictly controlled, this treatment promoted angiogenesis and blood perfusion, but it did not increase the risk of tumor metastasis [80, 81], suggesting that this strategy can safely address the challenge of pancreatic hypoperfusion for drug delivery.

(See figure on next page.)

Fig. 4 Nanoparticle optimization strategies used to reverse immunosuppression in the tumor microenvironment. a Some nanoparticles are used to target M2 TAMs. $\mathbf{b}$ Indoleamine 2,3-dioxygenase-1 (IDO1) siRNA-loaded lipid nanoparticles enhance immunogenic cell death (ICD) to promote immunogenic death of tumor cells. Reprinted by permission from [73]. c Co-encapsulation of the ICD inducer oxaliplatin and the IDO inhibitor indomethacin in mesoporous silica nanoparticles (MSNPs) coated with lipid bilayers. Reprinted by permission from [74]. DCs dendritic cells, CTLS cytotoxic T lymphocytes, OXA oxaliplatin, CRT calreticulin, HMGB-1 high-mobility group box 1, IND indoximod 
a

\begin{tabular}{|l|l|}
\hline & Strategy \\
\hline & $\begin{array}{l}\text { Porous silicon nanocarriers modified with the Ly6C antibody specifically } \\
\text { target Ly6C-overexpressing M2 tumor-associated macrophages (TAMs). }\end{array}$ \\
\hline
\end{tabular}

b

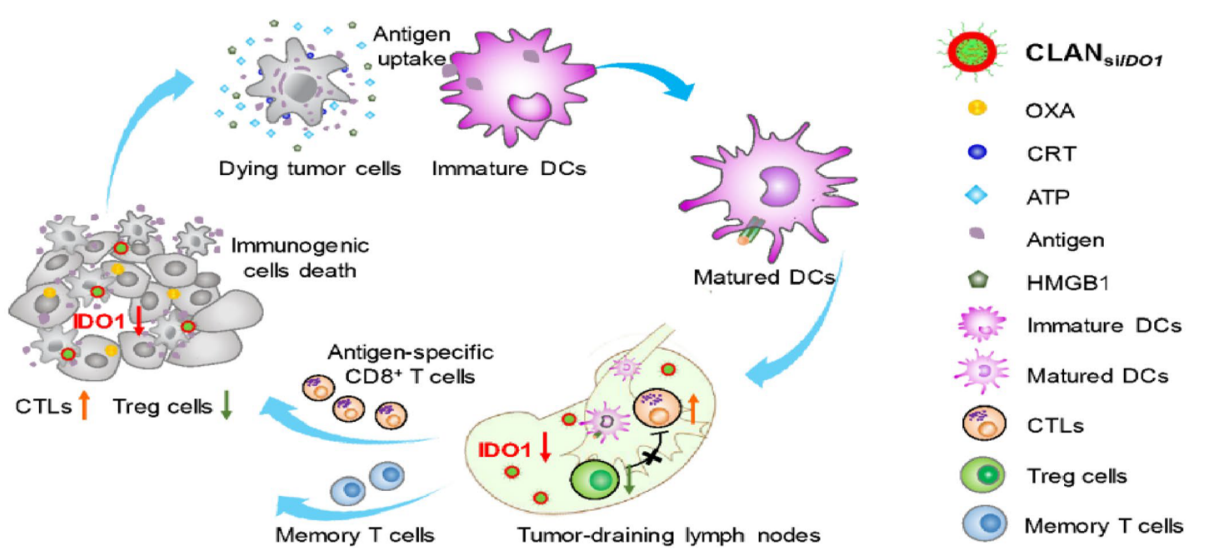

C
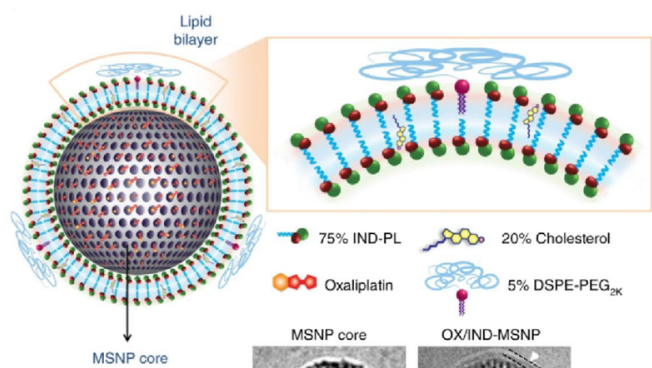

75\% IND-PL $5700020 \%$ Cholesterol

$\infty$ Oxaliplatin

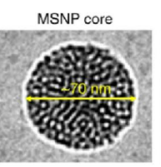

OXIND-MSNP

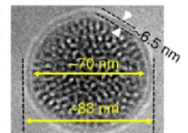

$100 \mathrm{~nm} \frac{1}{100 \mathrm{~nm}}$

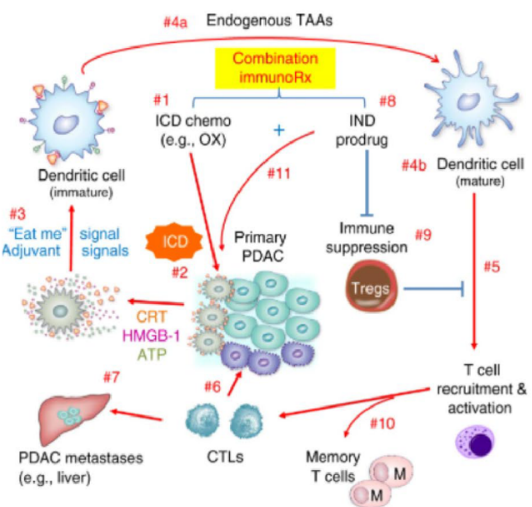

Fig. 4 (See legend on previous page.) 


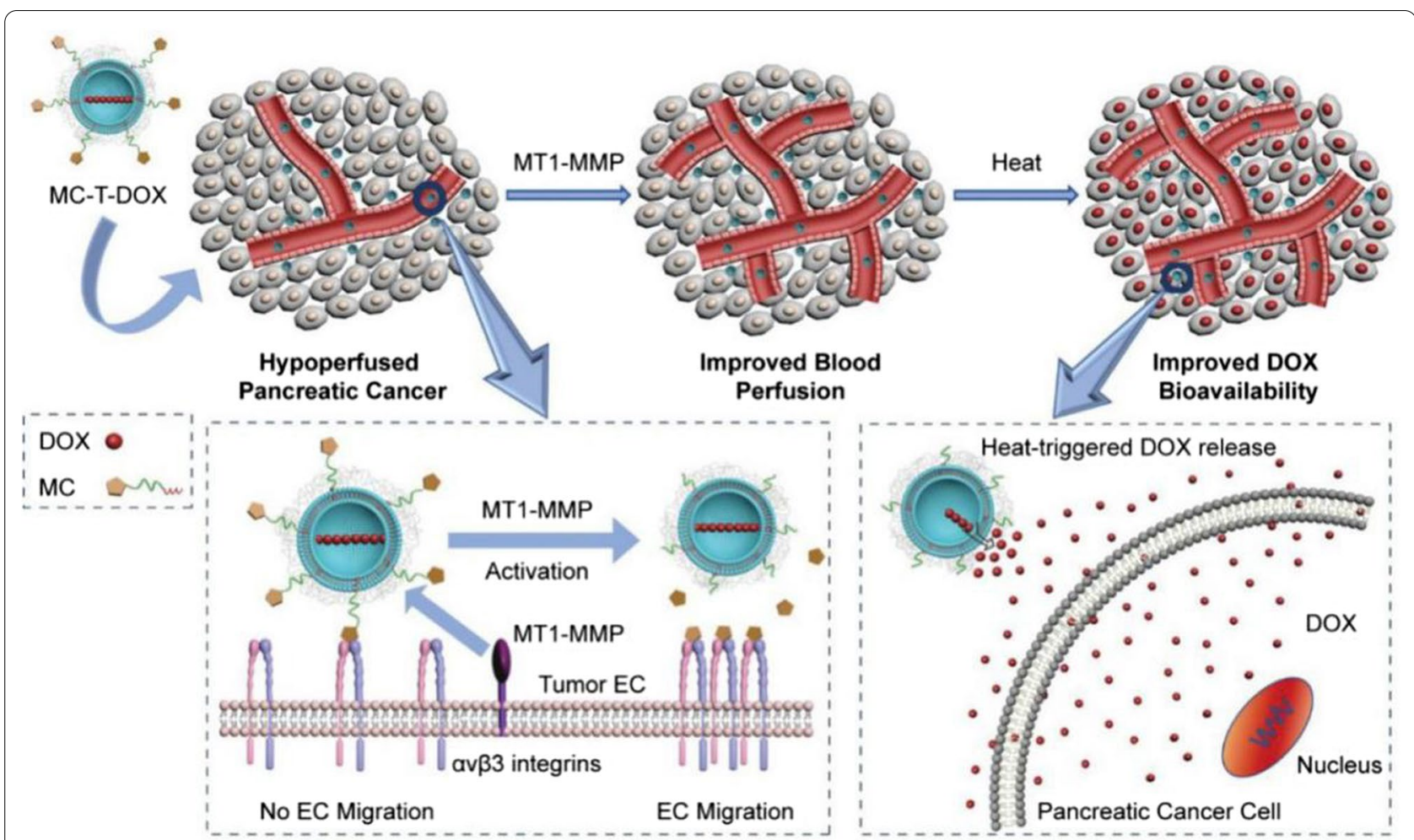

Fig. 5 Cilengitide encapsulated in a doxorubicin (DOX)-loaded thermosensitive liposome via a MT1-MMP-cleavable peptide improves tumor blood perfusion and drug delivery in pancreatic cancer. Cilengitide is released through MT1-MMP cleavage on tumor endothelial cells (ECS), promoting EC migration and angiogenesis. MT1-MMP, membrane type 1-matrix metalloproteinase; MC, MT1-MMP-activated cilengitide. Reprinted by permission from [79]

\section{Hypoxia}

The highly dense ECM and sparse vascular system in pancreatic cancer limit the delivery of oxygen to the tumor site. Pancreatic cancer cells adapt to the low oxygen levels, becoming invasive and drug-resistant and metastasizing even at early stages of tumor development [82-85]. Therefore, current research targeting hypoxia in pancreatic cancer focuses on (1) inhibiting the tumor hypoxic reaction, (2) transporting oxygen to the tumor, or (3) creating trigger release conditions in hypoxic tissues (Fig. 6).

\section{Inhibit the tumor hypoxic reaction}

Hypoxia-inducible factor- $1 \alpha$ (HIF- $1 \alpha)$ is considered the most promising target in the hypoxia signaling pathway to control cell metabolism, migration, and apoptosis, as well as regulate the transcription of angiogenesis growth factors [86, 87]. Therefore, the HIF-1 $\alpha$ inhibitor YC-1 was loaded into transferrin receptor-1-targeting liposomes for delivery to pancreatic tumors to improve hypoxia and anti-tumor effects [88]. However, selective gene silencing has proven more effective than chemical inhibition of HIF-1 $\alpha$ [89]. For example, negatively charged HIF-1 $\alpha$ siRNA (si-HIF1 $\alpha$ ) was adsorbed on the surface of a cationic $\varepsilon$-polylysine co-polymer by electrostatic interaction, while gemcitabine was encapsulated in its hydrophilic core [90]. The resulting copolymer was then coated with a PEGylated lipid membrane to prevent degradation of the anti-HIF1 $\alpha$ siRNA in serum. In vitro and in vivo experiments showed that HIF-1 $\alpha$ transcription was inhibited, the levels of hypoxia-induced factors were reduced, and the anti-tumor effect of gemcitabine was enhanced. However, the complex structure of lipid-polymer hybrid NPs did not allow the control of their particle size, limiting their application only to passive targeting [91]. Therefore, gemcitabine and anti-HIF1 $\alpha$ siRNA were co-loaded into GE11 peptide-conjugated cationic liposomes in order to actively target the epidermal growth factor receptor, which is overexpressed on the surface of cancer cells [92]. Although this approach significantly enhanced targeting ability, anti-HIF $1 \alpha$ siRNA may be unstable on the particle surface, and the cationic liposomes themselves may be cytotoxic. 


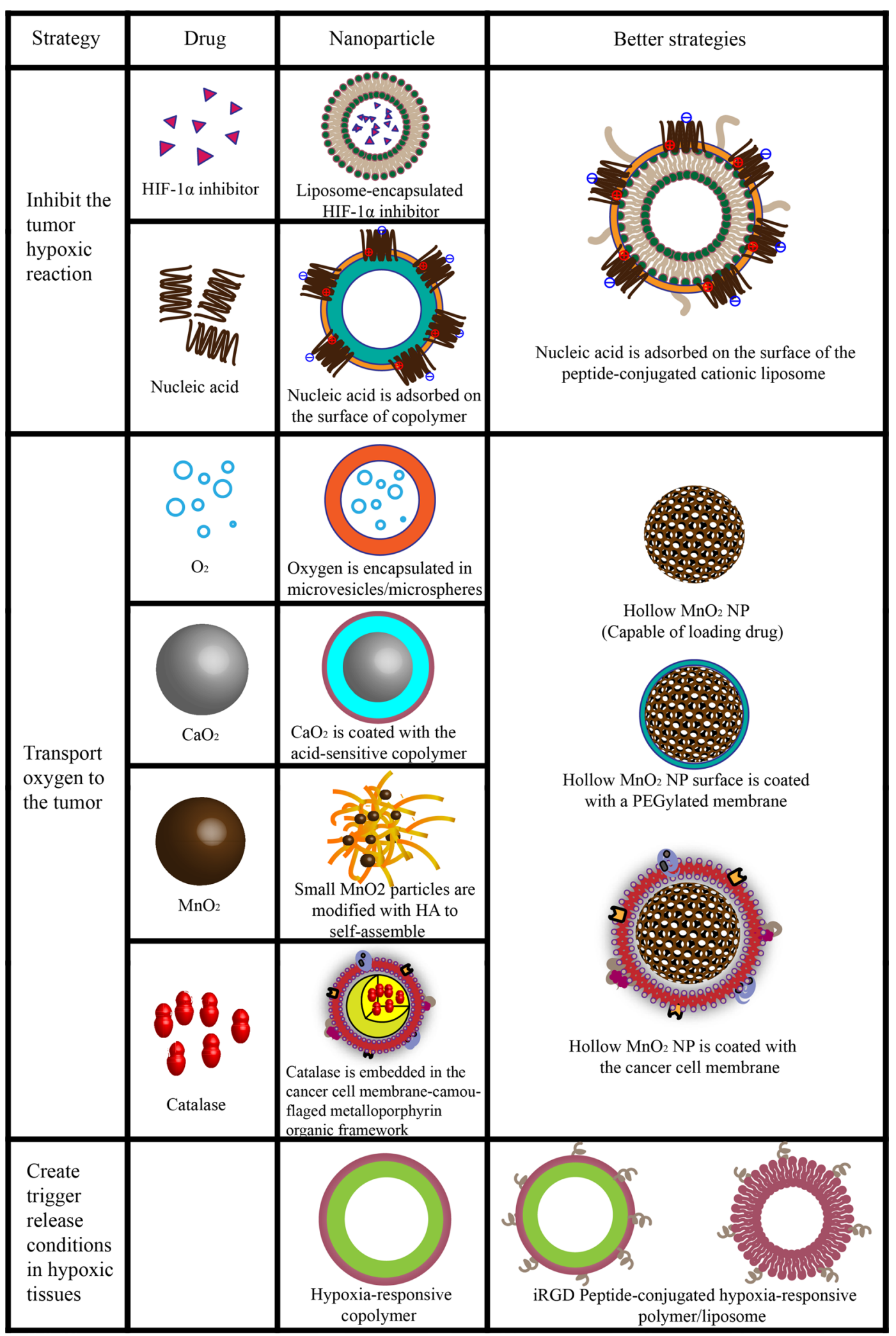

Fig. 6 Nanoparticle-based strategies used to target hypoxia in pancreatic cancer 


\section{Transport oxygen to the tumor}

Oxygen transport is considered a more direct strategy for relieving hypoxia. For example, oxygen encapsulated in microvesicles/microspheres was directly delivered to tumor hypoxia sites using ultrahigh pressure [93]. However, the microvesicles are unstable, they cannot target specific tissues, and the amount of oxygen delivered is difficult to control. Thus, this approach is mostly used in combination with other strategies, especially oxygen-consuming therapies such as collaborative acoustic power and photodynamic therapy [94-96].

Oxygen production based on the special conditions of the TME has also been reported as an effective approach. For example, NPs containing $\mathrm{CaO}_{2}$ and coated with acidsensitive methacrylate copolymers were prepared to control oxygen production [97]. Although the coating protected NPs from decomposition in the circulation, they were destroyed by the acidity of the hypoxic TME. This exposed the $\mathrm{CaO}_{2}$, which reacted with water to generate oxygen and reduce tumor hypoxia.

Excess $\mathrm{H}_{2} \mathrm{O}_{2}$ in tumor cells is another important source of oxygen. $\mathrm{MnO}_{2}$ or catalase have been used to promote $\mathrm{H}_{2} \mathrm{O}_{2}$ decomposition and alleviate tumor hypoxia. Small $\mathrm{MnO}_{2}$ particles $(\sim 15 \mathrm{~nm})$ were modified with HA to selfassemble into larger NPs for $\mathrm{H}_{2} \mathrm{O}_{2}$ depletion and oxygen production at the tumor site [98]. The hollow $\mathrm{MnO}_{2}$ surface was also coated with a PEGylated membrane to prevent premature clearance from the circulation and to produce oxygen in cancer cells [99]. However, PEGylation was found to accelerate clearance from the blood [100], suggesting that a polymer coating may be more effective for such NPs [101]. $\mathrm{MnO}_{2}$ NPs coated with cancer cell membrane proved to be more effective in targeting cancer cells, and the masking membrane allowed NPs to target tumor cells via specific plasma membrane proteins and homotypic adhesion [102]. Similarly, glucose oxidase and catalase were embedded in the cancer cell membrane-camouflaged metalloporphyrin organic framework of PCN-224 to catalyze $\mathrm{H}_{2} \mathrm{O}_{2}$-mediated oxygen production and synergistically enhance photodynamic therapy [103].

\section{Create trigger release conditions in hypoxic tissues}

The third hypoxia-targeting strategy involves the generation of trigger release conditions in hypoxic tissues. Hypoxia can be exploited as a triggering condition to induce drug release specifically in tumor tissues, while preventing drug delivery in normoxic tissues and therefore side effects $[104,105]$. For example, $90 \%$ of gemcitabine and erlotinib encapsulated in a hypoxia-responsive diblock copolymer poly(lactic acid)-azobenzenepoly(ethylene glycol) NP was released within $50 \mathrm{~min}$ under anoxic conditions, in contrast to no release under normal conditions [106]. The hypoxic area is located deep in the tumor tissue, close to the tumor core, where blood vessels are sparse, the impact of matrix accumulation is strong, and the degree of hypoxia is high [107]. Therefore, the modification of hypoxia-responsive NPs has been considered as a promising strategy to improve drug accumulation at the tumor core. For example, iRGD peptide-conjugated hypoxia-responsive liposomes were prepared, as the iRGD peptide can interact with integrins and neuropilin receptors overexpressed in tumor tissues [108]. This approach increased drug release in the deep hypoxic region of the pancreatic tumor. The iRGD peptide was also used to optimize hypoxia-responsive polymers [109].

\section{Challenges and potential problems}

TME is undeniably critical to the progression, invasion, and metastasis of pancreatic cancer, acting as a solid shield that protects the cancer cells at all-time and seriously impedes treatment progress. Nanobiotechnology is an emerging science in biomedical engineering [20], offering an opportunity to use NPs to target TME and treat cancer, which is like a sharp spear that can break the TME. Although some problems can be settled by NPs, still many challenges and potential problems we need to face.

The size of NPs is a significant factor affecting drug delivery and penetration in the stroma. Larger-sized NPs can gather around the tumor due to the prolonged circulation time, while the TME of pancreatic cancer has a denser stroma than other tumors, limiting their penetration into tumor. Oppositely, smaller-sized NPs can easily pass through it but are easily captured by mononuclear phagocyte system (MPS) [56]. Furthermore, surface charge properties of NPs are another factor that positively charged NPs are more likely to be uptake by cells than negatively charged ones, while negatively charged NPs have better stealth effect. As previously reviewed, positively charged NPs have shown potential efficacy against pancreatic cancer metastasis and have a better ability to load nucleic acid drugs $[73,110]$. In addition, the EPR effect is not nearly as significant in human tumors as it is in animal models [111], and no biomarkers specific for pancreatic cancer, which makes it hard to design advanced receptors and ligands for NPs in TME. When designing NPs targeting TME, these challenges should be addressed first.

Strategies for ECM have focused on ablating its components to break down barriers that prevent the diffusion of NPs through bioactive substances such as enzymes and peptides. Nevertheless, TME is a highly coordinated system, and this would destroy the balance with unpredictable adverse effects, such as increased tumor migration 
and metastasis. In addition, regulation of ECM by NPs with enzyme is mostly used to promote drug delivery, which is far from plenty for treatment. Therefore, NPsbased systems need to add extra drugs, which creates a perplexing problem that how to precisely control the proportion of two cargoes in the same NPs. On the one hand, excessive enzyme may lead to some unnecessary risks and affect the loading of anti-cancer drugs. On the other hand, small amounts of the enzyme do not show enough effects, and enzyme activity is more affected by anti-cancer drugs. Meanwhile, the combination of enzymes and NPs also needs to be taken into consideration. To this end, ECM modulation strategies by NPs should be carefully formulated and evaluated. Significantly different from ECM modulation strategies, unique angiogenesis therapy based on NPs for regulating TME of pancreatic cancer has been demonstrated to be effective [79]. Without destroying the original stroma, the composition of TME was adjusted to promote the penetration of NPs. Unfortunately, the number of studies reported in this field is extremely limited, possibly because there are some undiscovered potential risks.

Currently, the combination strategy tends to regulate the immune microenvironment [112]. However, the immune microenvironment in pancreatic cancer is a complex and sophisticated system. Our understanding of its components and complex interactions is far from enough so that even if different combination strategies are used, it is still problematic to choose a tailored solution. In addition, most of the oxygen self-production systems based on NPs need to confront the same challenge, namely sustainability of oxygen production. Stable and lasting oxygen release can better inhibit the expression of various hypoxia factors and play a role in adjuvant therapy, but this also appears to have not been noticeably resolved. Notably, human tumors which have many mutations are heterogeneous compared to animal, and TME varies from patient to patient [113]. Even though animal models can provide some valuable preclinical data, it does not mean they can be well explained and applied to human patients. Therefore, in order to better design and evaluate the nano-delivery system, it is imperative to build animal models that are more similar to human pancreatic tumors.

\section{Conclusions and outlook}

In recent decades, research on NPs for tumor cells has been approaching saturation but has not shown impressive efficacy. Regulation of TME based on nano-delivery system has brought a glimmer of hope for the treatment of pancreatic cancer. In this review, we discussed the regulation of pancreatic cancer microenvironment by nano-delivery systems, including remodeling ECM, targeting CAFs, regulating the immune microenvironment, promoting angiogenesis, and improving hypoxia condition. To sum up, although there are still some problems to be considered in the regulation of TME by NPs, such as the physicochemical properties of NPs, the heterogeneity of TME, and some potential therapeutic risks, it is still a promising research direction to improve drug delivery and tumor treatment effect. Furthermore, cancer cells and TME are a complete and complex community, and multifunctional NPs targeting both TME and cancer cells might be useful. In the end, we expect that continuing efforts to drive the development of new NPs-based therapies and strategies to target and reshape TME that will provide more effective direct or adjuvant treatments against pancreatic cancer.

\section{Abbreviations}

BCl2: B-cell lymphoma-2; BEZ 235: 2-Methyl-2-[4-(3-methyl-2-oxo-8-quinolin3-yl-2,3-dihydro-imidazo[4,5-c]quinolin-1-yl)-phenyl]-propionitrile; CAFs: Cancer-associated fibroblasts; $\mathrm{CaO}_{2}$ : Calcium peroxide; CAP: Cleavable amphiphilic peptide; CREKA: Cys-Arg-Glu-Lys-Ala; CRT: Calreticulin; CSF-1/ CSF-1R: Colony stimulating factor-1/colony stimulating factor-1 receptor; CTLs: Cytotoxic T lymphocytes; DCs: Dendritic cells; DOX: Doxorubicin; ECM: Extracellular matrix; ECs: Endothelial cells; FAP-a: Fibroblast-activated protein-a; GE11: A dodecapeptide (YHWYGYTPQNVI); GNPs: Gold nanoparticles; $\mathrm{H}_{2} \mathrm{O}_{2}$ : Hydrogen peroxide; HA: Hyaluronic acid; HAase: Hyaluronidase; HER2: Human epidermalgrowth factor receptor-2; HIF-1a: Hypoxia-inducible factor-1a; HMGB-1: High-mobility group box 1; HSA: Human serum albumin; ICD: Immunogenic cell death; IDO: Indoleamine 2,3-dioxygenase; IL: Interleukin; IND: Indoximod; iRGD: Cyclo(Cys-Arg-Gly-Asp-Lys-Gly-Pro-Asp-Cys); MMPs: Matrix metalloproteinases; $\mathrm{MnO}_{2}$ : Manganese dioxide; MSNPs: Mesoporous silica nanoparticles; MT: Membrane type; NPs: Nanoparticles; OXA: Oxaliplatin; PCN-224: Porphyrin Zr metal-organic framework; PDAC: Pancreatic ductal adenocarcinoma; PEG: Polyethylene glycol; PEGPH20: PEGylated recombinant human hyaluronidase PH20; PI3K- $\gamma$ : Phosphatidylinositol-3 kinase- $\gamma$; PLGA: Poly(lactic-co-glycolic acid); ppp dsRNA: 5'-Triphosphate double-stranded RNA; PTX: Paclitaxel; rHuPH20: Recombinant human hyaluronidase PH20; SHH: Sonic hedgehog; si-HIF1a: HIF-1 a siRNA; TAMs: Tumor-associated macrophages; TGF- $\beta$ : Transforming growth factor- $\beta$; TME: Tumor microenvironment; TNF-a: Tumor necrosis factor-a; Tregs: Regulatory T cells; YC-1: 3-(5'-Hydroxymethyl-2'-furyl)-1-benzylindazole.

\section{Acknowledgements}

Not applicable.

Authors' contributions

$\mathrm{MJ}$ and DZ contributed equally in preparing the original draft of the manuscript. CZ and CL guided the entire study. All authors read and approved the final manuscript.

\section{Funding}

This work was supported by the Science and Technology Project of Luzhou Government (2019-JYJ-51, 2019).

Availability of data and materials Not applicable.

\section{Declarations}

Ethics approval and consent to participate Not applicable. 


\section{Consent for publication}

We give our consent for the manuscript to be published in Journal of Nanobiotechnology.

\section{Competing interests}

The authors declare that they have no competing interests.

\section{Author details}

'Department of Pharmaceutical Sciences, School of Pharmacy, Southwest Medical University, No.1, Section 1, Xianglin Road, Luzhou, Sichuan 646000, People's Republic of China. ${ }^{2}$ Department of Pharmacy of Traditional Chinese Medicine, School of Pharmacy, Southwest Medical University, Luzhou 646000, Sichuan, China. ${ }^{3}$ The Key Laboratory of Medical Electrophysiology of the Ministry of Education, Southwest Medical University, No.1, Section 1, Xianglin Road, Luzhou, Sichuan 646000, People's Republic of China.

Received: 8 September 2021 Accepted: 12 November 2021 Published online: 22 November 2021

\section{References}

1. Ilic M, Ilic I. Epidemiology of pancreatic cancer. World J Gastroenterol. 2016;22(44):9694-705

2. Ansari $D$, Tingstedt $B$, Andersson B, Holmquist F, Sturesson C, Williamsson C, et al. Pancreatic cancer: yesterday, today and tomorrow. Future Oncol. 2016;12(16):1929-46.

3. Goral V. Pancreatic cancer: pathogenesis and diagnosis. Asian Pac J Cancer Prev. 2015;16(14):5619-24.

4. Lin QJ, Yang F, Jin C, Fu DL. Current status and progress of pancreatic cancer in China. World J Gastroenterol. 2015;21(26):7988-8003.

5. Carmicheal J, Patel A, Dalal V, Atri P, Dhaliwal AS, Wittel UA, et al. Elevating pancreatic cystic lesion stratification: current and future pancreatic cancer biomarker(s). Biochim Biophys Acta Rev Cancer. 2020;1873(1): 188318.

6. Adiseshaiah PP, Crist RM, Hook SS, McNeil SE. Nanomedicine strategies to overcome the pathophysiological barriers of pancreatic cancer. Nat Rev Clin Oncol. 2016;13(12):750-65.

7. Yang J, Ren B, Yang G, Wang H, Chen G, You L, et al. The enhancement of glycolysis regulates pancreatic cancer metastasis. Cell Mol Life Sci. 2020;77(2):305-21.

8. Wang S, Li Y, Xing C, Ding C, Zhang H, Chen L, et al. Tumor microenvironment in chemoresistance, metastasis and immunotherapy of pancreatic cancer. Am J Cancer Res. 2020;10(7):1937-53.

9. Karamitopoulou E. Tumour microenvironment of pancreatic cancer: immune landscape is dictated by molecular and histopathological features. Br J Cancer. 2019;121(1):5-14.

10. Nishida T, Yoshitomi H, Takano S, Kagawa S, Shimizu H, Ohtsuka M, et al. Low stromal area and high stromal microvessel density predict poor prognosis in pancreatic cancer. Pancreas. 2016:45(4):593-600.

11. Chen X, Zhou W, Liang C, Shi S, Yu X, Chen Q, et al. Codelivery nanosystem targeting the deep microenvironment of pancreatic cancer. Nano Lett. 2019;19(6):3527-34.

12. Ho WJ, Jaffee EM, Zheng L. The tumour microenvironment in pancreatic cancer-clinical challenges and opportunities. Nat Rev Clin Oncol. 2020;17(9):527-40.

13. Kokkinos J, Ignacio RMC, Sharbeen G, Boyer C, Gonzales-Aloy E, Goldstein $D$, et al. Targeting the undruggable in pancreatic cancer using nano-based gene silencing drugs. Biomaterials. 2020;240: 119742.

14. Wu J, Liang C, Chen M, Su W. Association between tumor-stroma ratio and prognosis in solid tumor patients: a systematic review and metaanalysis. Oncotarget. 2016;7(42):68954-65.

15. Li S, Xu HX, Wu CT, Wang WQ, Jin W, Gao HL, et al. Angiogenesis in pancreatic cancer: current research status and clinical implications. Angiogenesis. 2019;22(1):15-36.

16. Selvanesan BC, Meena K, Beck A, Meheus L, Lara O, Rooman I, et al. Nicotinamide combined with gemcitabine is an immunomodulatory therapy that restrains pancreatic cancer in mice. J Immunother Cancer. 2020;8(2): e001250.

17. Rocha FG. Landmark series: immunotherapy and targeted therapy for pancreatic cancer. Ann Surg Oncol. 2021;28(3):1400-6.
18. Magri A, Baveloni FG, de Camargo BAF, Chorilli M. The emerging landscapes to drug delivery systems for the treatment of pancreatic cancer. Curr Med Chem. 2021;28(26):5411-30.

19. Baetke SC, Lammers T, Kiessling F. Applications of NPs for diagnosis and therapy of cancer. Br J Radiol. 2015;88(1054):20150207.

20. Ashfaq UA, Riaz M, Yasmeen E, Yousaf MZ. Recent advances in nanoparticle-based targeted drug-delivery systems against cancer and role of tumor microenvironment. Crit Rev Ther Drug Carrier Syst. 2017;34(4):317-53.

21. Yang $\mathrm{S}, \mathrm{GaO} H$. NPs for modulating tumor microenvironment to improve drug delivery and tumor therapy. Pharmacol Res. 2017; 126:97-108.

22. Hessmann E, Buchholz SM, Demir IE, Singh SK, Gress TM, Ellenrieder V, et al. Microenvironmental determinants of pancreatic cancer. Physiol Rev. 2020;100(4):1707-51.

23. Neesse A, Bauer CA, Öhlund D, Lauth M, Buchholz M, Michl P, et al. Stromal biology and therapy in pancreatic cancer: ready for clinical translation? Gut. 2019;68(1):159-71.

24. Hosein AN, Brekken RA, Maitra A. Pancreatic cancer stroma: an update on therapeutic targeting strategies. Nat Rev Gastroenterol Hepatol. 2020;17(8):487-505.

25. Cox TR. The matrix in cancer. Nat Rev Cancer. 2021;21(4):217-38.

26. Wohlrab J, Wohlrab D, Wohlrab L, Wohlrab C, Wohlrab A. Use of hyaluronidase for pharmacokinetic increase in bioavailability of intracutaneously applied substances. Skin Pharmacol Physiol. 2014;27(5):276-82.

27. Zhong Y, Macgregor-Das A, Saunders T, Whittle MC, Makohon-Moore A, Kohutek ZA, et al. Mutant p53 together with TGF $\beta$ signaling influence organ-specific hematogenous colonization patterns of pancreatic cancer. Clin Cancer Res. 2017;23(6):1607-20.

28. Sidhu I, Barwe SP, Gopalakrishnapillai A. The extracellular matrix: a key player in the pathogenesis of hematologic malignancies. Blood Rev. 2021;48: 100787.

29. Jang M, An J, Oh SW, Lim JY, Kim J, Choi JK, et al. Matrix stiffness epigenetically regulates the oncogenic activation of the Yes-associated protein in gastric cancer. Nat Biomed Eng. 2021;5(1):114-23.

30. Piersma B, Hayward MK, Weaver VM. Fibrosis and cancer: a strained relationship. Biochim Biophys Acta Rev Cancer. 2020;1873(2): 188356.

31. Wilhelm S, Tavares AJ, Dai Q, Ohta S, Audet J, Dvorak HF, Chan WCW. Analysis of nanoparticle delivery to tumours. Nat Rev Mater. 2016. https://doi.org/10.1038/natrevmats.2016.14.

32. Korc M. Pancreatic cancer-associated stroma production. Am J Surg. 2007;194(4 Suppl):S84-6.

33. Girigoswami K, Saini D, Girigoswami A. Extracellular matrix remodeling and development of cancer. Stem Cell Rev Rep. 2021;17(3):739-47.

34. Doherty GJ, Tempero M, Corrie PG. HALO-109-301: a Phase III trial of PEGPH20 (with gemcitabine and nab-paclitaxel) in hyaluronic acid-high stage IV pancreatic cancer. Future Oncol. 2018;14(1):13-22.

35. Sohr S, Engeland K. RHAMM is differentially expressed in the cell cycle and downregulated by the tumor suppressor p53. Cell Cycle. 2008;7(21):3448-60.

36. Whatcott CJ, Diep CH, Jiang P, Watanabe A, LoBello J, Sima C, et al. Desmoplasia in primary tumors and metastatic lesions of pancreatic cancer. Clin Cancer Res. 2015;21(15):3561-8.

37. Jacobetz MA, Chan DS, Neesse A, Bapiro TE, Cook N, Frese KK, et al. Hyaluronan impairs vascular function and drug delivery in a mouse model of pancreatic cancer. Gut. 2013;62(1):112-20.

38. Zhou H, Fan Z, Deng J, Lemons PK, Arhontoulis DC, Bowne WB, et al. Hyaluronidase embedded in nanocarrier PEG shell for enhanced tumor penetration and highly efficient antitumor efficacy. Nano Lett. 2016;16(5):3268-77.

39. Chen D, Zhu X, Tao W, Kong Y, Huag Y, Zhang Y, et al. Regulation of pancreatic cancer microenvironment by an intelligent gemcitabine@ nanogel system via in vitro 3D model for promoting therapeutic efficiency. J Control Release. 2020;10(324):545-59.

40. Wang H, Han X, Dong Z, et al. Hyaluronidase with $\mathrm{pH}$-responsive dextran modification as an adjuvant nanomedicine for enhanced photodynamic-immunotherapy of cancer. Adv Funct Mater. 2019;29:1902440.

41. Weber GC, Buhren BA, Schrumpf H, Wohlrab J, Gerber PA. Clinical applications of hyaluronidase. Adv Exp Med Biol. 2019;1148:255-77.

42. Xu S, Xu H, Wang W, Li S, Li H, Li T, et al. The role of collagen in cancer: from bench to bedside. J Transl Med. 2019;17(1):309. 
43. Egeblad M, Rasch MG, Weaver VM. Dynamic interplay between the collagen scaffold and tumor evolution. Curr Opin Cell Biol. 2010;22(5):697-706

44. Chen IM, Willumsen N, Dehlendorff C, Johansen AZ, Jensen BV, Hansen $C P$, et al. Clinical value of serum hyaluronan and propeptide of type III collagen in patients with pancreatic cancer. Int J Cancer. 2020;146(10):2913-22.

45. Villegas MR, Baeza A, Usategui A, Ortiz-Romero PL, Pablos JL, Vallet-Regí M. Collagenase nanocapsules: an approach to fibrosis treatment. Acta Biomater. 2018;1(74):430-8.

46. Zinger A, Koren L, Adir O, Poley M, Alyan M, Yaari Z, et al. Collagenase NPs enhance the penetration of drugs into pancreatic tumors. ACS Nano. 2019;13(10):11008-21.

47. Liang C, Shi S, Meng Q, Liang D, Ji S, Zhang B, et al. Do anti-stroma therapies improve extrinsic resistance to increase the efficacy of gemcitabine in pancreatic cancer? Cell Mol Life Sci. 2018;75(6):1001-12.

48. Amoozgar Z, Goldberg MS. Surface modulation of polymeric nanocarriers enhances the stability and delivery of proteins and small molecules. Nanomedicine (Lond). 2017;12(7):729-43.

49. Pan A, Wang Z, Chen B, Dai W, Zhang H, He B, et al. Localized codelivery of collagenase and trastuzumab by thermosensitive hydrogels for enhanced antitumor efficacy in human breast xenograft. Drug Deliv. 2018;25(1):1495-503.

50. Dolor A, Szoka FC Jr. Digesting a path forward: the utility of collagenase tumor treatment for improved drug delivery. Mol Pharm. 2018;15(6):2069-83

51. Knapinska AM, Estrada CA, Fields GB. The roles of matrix metalloproteinases in pancreatic cancer. Prog Mol Biol Transl Sci. 2017;148:339-54.

52. Yin N, Yu H, Zhang X, Lv X. Enhancement of pancreatic cancer therapy efficacy by type-1 matrix metalloproteinase-functionalized NPs for the selective delivery of gemcitabine and erlotinib. Drug Des Devel Ther. 2020;23(14):4465-75.

53. Kulkarni PS, Haldar MK, Nahire RR, Katti P, Ambre AH, Muhonen WW, et al. Mmp-9 responsive PEG cleavable nanovesicles for efficient delivery of chemotherapeutics to pancreatic cancer. Mol Pharm. 2014;11(7):2390-9.

54. Melstrom LG, Salazar MD, Diamond DJ. The pancreatic cancer microenvironment: a true double agent. J Surg Oncol. 2017;116(1):7-15.

55. von Ahrens D, Bhagat TD, Nagrath D, Maitra A, Verma A. The role of stromal cancer-associated fibroblasts in pancreatic cancer. J Hematol Oncol. 2017;10(1):76.

56. Yu Q, Qiu Y, Li J, Tang X, Wang X, Cun X, et al. Targeting cancer-associated fibroblasts by dual-responsive lipid-albumin NPs to enhance drug perfusion for pancreatic tumor therapy. J Control Release. 2020;10(321):564-75.

57. Biffi G, Tuveson DA. Diversity and biology of cancer-associated fibroblasts. Physiol Rev. 2021;101(1):147-76.

58. Giorello MB, Borzone FR, Labovsky V, Piccioni FV, Chasseing NA. Cancerassociated fibroblasts in the breast tumor microenvironment. J Mammary Gland Biol Neoplasia. 2021;26(2):135-55.

59. Lakiotaki E, Sakellariou S, Evangelou K, Liapis G, Patsouris E, Delladetsima I. Vascular and ductal elastotic changes in pancreatic cancer. APMIS. 2016;124(3):181-7.

60. Pereira BA, Vennin C, Papanicolaou M, Chambers CR, Herrmann D, Morton JP, et al. CAF subpopulations: a new reservoir of stromal targets in pancreatic cancer. Trends Cancer. 2019;5(11):724-41.

61. Linares J, Marín-Jiménez JA, Badia-Ramentol J, Calon A. Determinants and functions of CAFs secretome during cancer progression and therapy. Front Cell Dev Biol. 2021;8: 621070.

62. Feng J, Xu M, Wang J, Zhou S, Liu Y, Liu S, et al. Sequential delivery of nanoformulated a-mangostin and triptolide overcomes permeation obstacles and improves therapeutic effects in pancreatic cancer. Biomaterials. 2020;241: 119907.

63. Jiang T, Zhang B, Zhang L, Wu X, Li H, Shen S, et al. Biomimetic NPS delivered hedgehog pathway inhibitor to modify tumour microenvironment and improved chemotherapy for pancreatic carcinoma. Artif Cells Nanomed Biotechnol. 2018;46(sup 1):1088-101.

64. Zhao J, Wang H, Hsiao CH, Chow DS, Koay EJ, Kang Y, et al. Simultaneous inhibition of hedgehog signaling and tumor proliferation remodels stroma and enhances pancreatic cancer therapy. Biomaterials. 2018;159:215-28.
65. Hossen MN, Rao G, Dey A, Robertson JD, Bhattacharya R, Mukherjee P. Gold nanoparticle transforms activated cancer-associated fibroblasts to quiescence. ACS Appl Mater Interfaces. 2019;11(29):26060-8.

66. Cheng N, Bai X, Shu Y, Ahmad O, Shen P. Targeting tumor-associated macrophages as an antitumor strategy. Biochem Pharmacol. 2021;183: 114354.

67. Peng H, Wang JH, Guo F, Zhu FF, Wen ZJ, Zhong HJ, et al. Legumain protease-activated tuftsin-functionalized nanoparticles for dualtargeting TAMs and cancer chemotherapy. Colloids Surf B Biointerfaces. 2021;197: 111442.

68. Cullis J, Siolas D, Avanzi A, Barui S, Maitra A, Bar-Sagi D. Macropinocytosis of nab-paclitaxel drives macrophage activation in pancreatic cancer. Cancer Immunol Res. 2017;5(3):182-90.

69. Wang N, Wang S, Wang X, Zheng Y, Yang B, Zhang J, et al. Research trends in pharmacological modulation of tumor-associated macrophages. Clin Transl Med. 2021;11(1): e288.

70. Yokoi K, Godin B, Oborn CJ, Alexander JF, Liu X, Fidler IJ, et al. Porous silicon nanocarriers for dual targeting tumor associated endothelial cells and macrophages in stroma of orthotopic human pancreatic cancers. Cancer Lett. 2013;334(2):319-27.

71. Li M, Li M, Yang Y, Liu Y, Xie H, Yu Q, et al. Remodeling tumor immune microenvironment via targeted blockade of PI3K- $\gamma$ and CSF-1/CSF-1R pathways in tumor associated macrophages for pancreatic cancer therapy. J Control Release. 2020;10(321):23-35.

72. Das M, Shen L, Liu Q, Goodwin TJ, Huang L. Nanoparticle delivery of RIG-I agonist enables effective and safe adjuvant therapy in pancreatic cancer. Mol Ther. 2019;27(3):507-17.

73. Huang H, Jiang CT, Shen S, Liu A, Gan YJ, Tong QS, et al. Nanoenabled reversal of IDO1-mediated immunosuppression synergizes with immunogenic chemotherapy for improved cancer therapy. Nano Lett. 2019;19(8):5356-65.

74. Lu J, Liu X, Liao YP, Salazar F, Sun B, Jiang W, et al. Nano-enabled pancreas cancer immunotherapy using immunogenic cell death and reversing immunosuppression. Nat Commun. 2017;8(1):1811.

75. Reynolds AR, Hart IR, Watson AR, Welti JC, Silva RG, Robinson SD, et al. Stimulation of tumor growth and angiogenesis by low concentrations of RGD-mimetic integrin inhibitors. Nat Med. 2009;15(4):392-400.

76. Brooks PC, Montgomery AM, Rosenfeld M, Reisfeld RA, Hu T, Klier $G$, et al. Integrin alpha $v$ beta 3 antagonists promote tumor regression by inducing apoptosis of angiogenic blood vessels. Cell. 1994;79(7):1157-64

77. Reardon DA, Neyns B, Weller M, Tonn JC, Nabors LB, Stupp R. Cilengitide: an RGD pentapeptide av $\beta 3$ and av $\beta 5$ integrin inhibitor in development for glioblastoma and other malignancies. Future Oncol. 2011;7(3):339-54

78. Mas-Moruno C, Rechenmacher F, Kessler H. Cilengitide: the first anti-angiogenic small molecule drug candidate design, synthesis and clinical evaluation. Anticancer Agents Med Chem. 2010;10(10):753-68.

79. Wei Y, Song S, Duan N, Wang F, Wang Y, Yang Y, et al. MT1-MMPactivated liposomes to improve tumor blood perfusion and drug delivery for enhanced pancreatic cancer therapy. Adv Sci (Weinh). 2020;7(17):1902746.

80. Özdemir BC, Pentcheva-Hoang T, Carstens JL, Zheng X, Wu CC, Simpson TR, et al. Depletion of carcinoma-associated fibroblasts and fibrosis induces immunosuppression and accelerates pancreas cancer with reduced survival. Cancer Cell. 2014;25(6):719-34.

81. Rhim AD, Oberstein PE, Thomas DH, Mirek ET, Palermo CF, Sastra SA et al. Stromal elements act to restrain, rather than support, pancreatic ductal adenocarcinoma. Cancer Cell. 2014;25(6):735-47.

82. Erkan M, Kurtoglu M, Kleeff J. The role of hypoxia in pancreatic cancer: a potential therapeutic target? Expert Rev Gastroenterol Hepatol. 2016;10(3):301-16.

83. Hao X, Ren Y, Feng M, Wang Q, Wang Y. Metabolic reprogramming due to hypoxia in pancreatic cancer: Implications for tumor formation, immunity, and more. Biomed Pharmacother. 2021;141: 111798.

84. Tao J, Yang G, Zhou W, Qiu J, Chen G, Luo W, et al. Targeting hypoxic tumor microenvironment in pancreatic cancer. J Hematol Oncol. 2021;14(1):14

85. Donato C, Kunz L, Castro-Giner F, Paasinen-Sohns A, Strittmatter K, Szczerba BM, et al. Hypoxia triggers the intravasation of clustered circulating tumor cells. Cell Rep. 2020;32(10): 108105. 
86. Feig C, Gopinathan A, Neesse A, Chan DS, Cook N, Tuveson DA. The pancreas cancer microenvironment. Clin Cancer Res. 2012;18(16):4266-76.

87. Mu R, Zou YK, Tu K, Wang DB, Tang D, Yu Z, et al. Hypoxia promotes pancreatic cancer cell dedifferentiation to stem-like cell phenotypes with high tumorigenic potential by the HIF-1a/notch signaling pathway. Pancreas. 2021;50(5):756-65.

88. Lang J, Zhao X, Wang X, Zhao Y, Li Y, Zhao R, et al. Targeted co-delivery of the iron chelator deferoxamine and a HIF1a inhibitor impairs pancreatic tumor growth. ACS Nano. 2019;13(2):2176-89.

89. Mizuno T, Nagao M, Yamada Y, Narikiyo M, Ueno M, Miyagishi M, et al. Small interfering RNA expression vector targeting hypoxia-inducible factor 1 alpha inhibits tumor growth in hepatobiliary and pancreatic cancers. Cancer Gene Ther. 2006;13(2):131-40.

90. Zhao X, Li F, Li Y, Wang H, Ren H, Chen J, et al. Co-delivery of HIF1a siRNA and gemcitabine via biocompatible lipid-polymer hybrid NPs for effective treatment of pancreatic cancer. Biomaterials. 2015;46:13-25.

91. Danaei M, Dehghankhold M, Ataei S, Hasanzadeh Davarani F, Javanmard R, Dokhani A, et al. Impact of particle size and polydispersity index on the clinical applications of lipidic nanocarrier systems. Pharmaceutics. 2018;10(2):57.

92. Lin C, Hu Z, Yuan G, Su H, Zeng Y, Guo Z, et al. HIF1a-siRNA and gemcitabine combination-based GE-11 peptide antibody-targeted nanomedicine for enhanced therapeutic efficacy in pancreatic cancers. J Drug Target. 2019;27(7):797-805.

93. McEwan C, Owen J, Stride E, Fowley C, Nesbitt H, Cochrane D, et al. Oxygen carrying microbubbles for enhanced sonodynamic therapy of hypoxic tumours. J Control Release. 2015;10(203):51-6.

94. Sheng Y, Beguin E, Nesbitt H, Kamila S, Owen J, Barnsley LC, et al. Magnetically responsive microbubbles as delivery vehicles for targeted sonodynamic and antimetabolite therapy of pancreatic cancer. J Control Release. 2017;28(262):192-200

95. McEwan C, Kamila S, Owen J, Nesbitt H, Callan B, Borden M, et al. Combined sonodynamic and antimetabolite therapy for the improved treatment of pancreatic cancer using oxygen loaded microbubbles as a delivery vehicle. Biomaterials. 2016;80:20-32.

96. Chen J, Luo H, Liu Y, Zhang W, Li H, Luo T, et al. Oxygen-selfproduced nanoplatform for relieving hypoxia and breaking resistance to sonodynamic treatment of pancreatic cancer. ACS Nano. 2017;11(12):12849-62.

97. Sheng Y, Nesbitt H, Callan B, Taylor MA, Love M, McHale AP, et al. Oxygen generating NPs for improved photodynamic therapy of hypoxic tumours. J Control Release. 2017;28(264):333-40.

98. Song M, Liu T, Shi C, Zhang X, Chen X. Bioconjugated manganese dioxide NPs enhance chemotherapy response by priming tumor-associated macrophages toward M1-like phenotype and attenuating tumor hypoxia. ACS Nano. 2016;10(1):633-47.

99. Yang G, Xu L, Chao Y, Xu J, Sun X, Wu Y, et al. Hollow $\mathrm{MnO}_{2}$ as a tumormicroenvironment-responsive biodegradable nano-platform for combination therapy favoring antitumor immune responses. Nat Commun. 2017;8(1):902
100. Abu Lila AS, Kiwada $\mathrm{H}$, Ishida T The accelerated blood clearance (ABC) phenomenon: clinical challenge and approaches to manage. J Control Release. 2013:172(1):38-47.

101. Shin SW, Jung W, Choi C, Kim SY, Son A, Kim H, et al. Fucoidan-manganese dioxide NPs potentiate radiation therapy by co-targeting tumor hypoxia and angiogenesis. Mar Drugs. 2018;16(12):510.

102. Pan W, Ge Y, Yu Z, Zhou P, Cui B, Li N, et al. A cancer cell membraneencapsulated $\mathrm{MnO}_{2}$ nanoreactor for combined photodynamic-starvation therapy. Chem Commun (Camb). 2019;55(35):5115-8.

103. Li SY, Cheng H, Xie BR, Qiu WX, Zeng JY, Li CX, et al. Cancer cell membrane camouflaged cascade bioreactor for cancer targeted starvation and photodynamic therapy. ACS Nano. 2017;11(7):7006-18.

104. Liang D, Miller GH, Tranmer GK. Hypoxia activated prodrugs: factors influencing design and development. Curr Med Chem. 2015;22(37):4313-25.

105. Gulfam M, Sahle FF, Lowe TL. Design strategies for chemical-stimuliresponsive programmable nanotherapeutics. Drug Discov Today. 2019:24(1):129-47.

106. Kulkarni P, Haldar MK, You S, Choi Y, Mallik S. Hypoxia-responsive polymersomes for drug delivery to hypoxic pancreatic cancer cells. Biomacromol. 2016;17(8):2507-13.

107. Graham K, Unger E. Overcoming tumor hypoxia as a barrier to radiotherapy, chemotherapy and immunotherapy in cancer treatment. Int J Nanomed. 2018:4(13):6049-58.

108. Kulkarni P, Haldar MK, Katti P, Dawes C, You S, Choi Y, et al. Hypoxia responsive, tumor penetrating lipid NPs for delivery of chemotherapeutics to pancreatic cancer cell spheroids. Bioconjug Chem. 2016;27(8):1830-8.

109. Confeld MI, Mamnoon B, Feng L, Jensen-Smith H, Ray P, Froberg J, et al. Targeting the tumor core: hypoxia-responsive NPs for the delivery of chemotherapy to pancreatic tumors. Mol Pharm. 2020;17(8):2849-63.

110. Ichihara H, Motomura M, Matsumoto Y. Therapeutic effects and anti-metastasis effects of cationic liposomes against pancreatic cancer metastasis in vitro and in vivo. Biochem Biophys Res Commun. 2019:511(3):504-9.

111. Lin G, Chen S, Mi P. Nanoparticles targeting and remodeling tumor microenvironment for cancer theranostics. J Biomed Nanotechnol. 2018;14(7):1189-207.

112. Galon J, Bruni D. Approaches to treat immune hot, altered and cold tumours with combination immunotherapies. Nat Rev Drug Discov. 2019;18(3):197-218.

113. Musetti S, Huang L. Nanoparticle-mediated remodeling of the tumor microenvironment to enhance immunotherapy. ACS Nano. 2018;12(12):11740-55.

\section{Publisher's Note}

Springer Nature remains neutral with regard to jurisdictional claims in published maps and institutional affiliations.
Ready to submit your research? Choose BMC and benefit from:

- fast, convenient online submission

- thorough peer review by experienced researchers in your field

- rapid publication on acceptance

- support for research data, including large and complex data types

- gold Open Access which fosters wider collaboration and increased citations

- maximum visibility for your research: over $100 \mathrm{M}$ website views per year

At $\mathrm{BMC}$, research is always in progress.

Learn more biomedcentral.com/submissions 